\title{
THE NOVEMBER MEETING IN AUBURN
}

The four hundred seventy-fourth meeting of the American Mathematical Society was held at Alabama Polytechnic Institute, Auburn, Alabama, on Friday and Saturday, November 23-24, 1951. The total attendance was 130 including the following 66 members of the Society:

O. R. Ainsworth, M. G. Boyce, A. T. Brauer, R. K. Butz, H. E. Campbell, A. C. Cohen, Haskell Cohen, R. M. Conkling, J. C. Currie, V. E. Dietrich, F. G. Dressel, B. M. Drucker, W. L. Duren, Patrick Du Val, J. C. Eaves, M. W. Effron, D. O. Ellis, M. E. Estill, Tomlinson Fort, H. K. Fulmer, Wallace Givens, D. B. Goodner, E. H. Hadloch, B. F. Hadnot, E. V. Haynsworth, Nickolas Heerema, Melvin Henriksen, G. B. Huff, Ernest Ikenberry, J. L. Kelley, R. J. Koch, F. W. Kokomoor, G. B. Lang, R. J. Levit, F. A. Lewis, C. W. McArthur, Nathaniel Macon, G. W. Medlin, E. P. Miles, Knox Millsaps, Benjamin Ernest Mitchell, Benjamin Evans Mitchell, J. D. Morales, D. R. Morrison, W. V. Parker, E. H. Pearson, W. D. Peeples, A. S. Rayl, Herbert Robbins, J. H. Roberts, F. V. Rohde, W. A. Rutledge, H. D. Sprinkle, H. S. Stanley, H. E. Taylor, J. M. Thomas, H. S. Thurston, G. J. Trammell, B. O. Van Hook, T. L. Wade, J. H. Wahab, W. M. Whyburn, Ernest Williams, G. N. Wollan, C. T. Yang, J. W. Young.

By invitation of the Committee to Select Hour Speakers for Southeastern Sectional Meetings, Professor H. E. Robbins of the University of North Carolina and Professor J. L. Kelley of Tulane University addressed the Society. Professor Robbins' address, entitled Mathematical aspects of the sequential design of experiments, was delivered at 8:00 P.M. on Friday, with Professor W. V. Parker presiding. The address by Professor Kelley, entitled Duality in algebraic analysis, was given at 10:00 A.M. on Saturday, with Professor Tomlinson Fort presiding.

Four sessions for the presentation of contributed papers were held on Friday afternoon and Saturday morning. Presiding officers for these sessions were Professor J. H. Roberts, F. W. Kokomoor, T. L. Wade, and W. L. Duren.

President Ralph D. Draughon of Alabama Polytechnic Institute extended greetings at the opening of the evening session on Friday. All in attendance at the meetings were guests of the host institution and its Department of Mathematics at a reception and social hour which followed the hour address on Friday evening. A resolution expressing the appreciation of the Society to its hosts for the meeting was offered by Professor Tomlinson Fort and unanimously adopted.

Abstracts of the papers presented follow below. Abstracts whose number are followed by the letter " $t$ " were presented by title. Paper number 59 was read by Professor Dressel. Professor Strother was 
introduced by Professor Wallace, Mr. Davis by Professor Brauer, Mr. Newton by Professor Fort, Mr. J. W. Ellis by Professor Pettis, and Professor Willoughby by Professor Whyburn.

\section{Algebra AND Theory OF Numbers}

35. A. T. Brauer: Limits for the characteristic roots of a matrix. IV. Applications to stochastic matrices.

It is well known that the characteristic roots of a stochastic matrix $A=\left(a_{\kappa \lambda}\right)$ lie in the circle $|z| \leqq 1$. The point $z=1$ is always a characteristic root. If $A$ is unreduced, then 1 is a simple root. Let $a_{r r}$ and $a_{s s}$ be the smallest elements of the main diagonal of $A$ and $a_{r r} \leqq a_{s s}$. It was proved by Fréchet (Publications de la Faculté des Sciences de l'Université Masaryk no. 178 (1933) pp. 1-24) that the characteristic roots of $A$ lie in the circle $\left|z-a_{r r}\right| \leqq 1-a_{r r}$. In this paper it is proved that the circle can be replaced by the oval of Cassini $\left|z-a_{r r}\right|\left|z-a_{s s}\right| \leqq\left(1-a_{r r}\right)\left(1-a_{s s}\right)$. Let $h_{1}, h_{2}, \cdots, h_{n}$ be arbitrary numbers and $B$ the matrix $\left(a_{k \lambda}-h_{\lambda}\right)$. Assume that $A$ is unreduced. It is shown that the roots of $A$ coincide with those of $B$ and have the same multiplicity for $A$ and $B$, except that the simple root 1 of $A$ is replaced by $1-h_{1}-h_{2}-\cdots-h_{n}$. Therefore it is of ten possible to obtain better bounds for the nontrivial characteristic roots of $A$ by applying the results of the first 3 parts of this paper (Duke Math. J. vol. 13 (1946) pp. 387-395; vol. 14 (1947) pp. 21-26; vol. 15 (1948) pp. 871-877) to the matrix $B$ instead of $A$. (Received October 9, 1951.)

\section{6t. Leonard Carlitz: A theorem of Glaisher.}

Glaisher (Quarterly Journal of Mathematics vol. 31 (1900) p. 325) showed that if $(x+1)(x+2) \cdots(x+p-1)=x^{p-1}+A_{1} x^{p-2}+\cdots+A_{p-1}$, where $p$ is a prime $>3$, then for $1 \leqq t<2^{-1}(p-1), A_{2 t} / p \equiv-B_{2 t} / 2 p, A_{2 t+1} / p^{2} \equiv(2 t+1) B_{2 t} / 4 t(\bmod p)$. In the present paper the author (1) makes several new applications of Glaisher's formulas, (2) shows that $A_{2 t+1} \equiv-((p-2 t-1) / 4 t) p^{2} B_{2 t}-((2 t+1) / 2 t) p^{3} \sum_{k=1}^{t=1}(1 / 4 k) B_{2 k} B_{2 t-2 k}$ $\left(\bmod p^{4}\right)$, and $(3)$ extends Glaisher's theorem to more general sequences. The generalization depends on the relation of the $A_{r}$ to Bernoulli number of higher order. (Received October 8, 1951.)

\section{7t. Leonard Carlitz: Congruences for the coefficients of hyperelliptic functions.}

In a previous paper (Duke Math. J. vol. 16 (1949) pp. 297-302) the writer showed that if $f(x)=\operatorname{sn}\left(x, k^{2}\right)=\sum_{1}^{\infty} a_{i} x^{i} / i !$, and $f^{m}(x)=\sum_{i=m}^{\infty} a_{i}^{(m)} x^{i} / i !, a_{i}^{(1)}=a_{i}$, then $\sum_{i=0}^{r}(-1)^{i} C_{r, i} a_{p}^{r-i} a_{m+r+i(p-1)}^{(h)} \equiv 0\left(\bmod p^{r}\right)$ for $m \geqq 0, r \geqq 1, h \geqq 1$, and $l=k^{2}$ integral $(\bmod p)$. In the present paper similar results are obtained for the coefficients of hyperelliptic functions and more generally for functions $g(x)$ with inverse of the type $\sum_{1}^{\infty} e_{m} x^{m} / m$; however the modulus is now only $p^{s}$, where $s=\left[2^{-1}(r+k+1)\right]$, where $k=[h / p]$. Like results are also obtained for the coefficients of $x / g(x)$. (Received October 8, 1951.)

38t. Leonard Carlitz: Some congruences for the Bernoulli numbers.

Vandiver (Duke Math. J. vol. 5 (1939) pp. 548-551) proved that $\sum_{i=0}^{r}(-1)^{i} C_{r, i} B_{(i+k)(p-1)} \equiv 0\left(\bmod p^{r-1}\right)$ for $p>2, k>0, r>0, k+r<p-1$. In the present paper it is shown by a somewhat different method that $\sum_{i=0}^{r}(-1)^{i} C_{r, i} B_{m+i(p-1)}$ 
$\equiv 0\left(\bmod p^{r-1}\right)$, where $m=(h p+k)(p-1), 1 \leqq r<k+r<p$. It is also shown that $\sum_{i=0}^{r}(-1)^{r} C_{r, i}\left(B_{(i+1)(p-1)}+1 / p-1\right) /(i+1) \equiv 0\left(\bmod p^{r}\right)$ for $r<p-1$, and that $B_{m(p-1)}+1 / p-1 \equiv 0\left(\bmod p^{r}\right)$ for $p^{r} \mid m$. (Received October 8,1951 .)

39. J. C. Currie: Canonical forms for the Lorentz-Hermitian matrix. Preliminary report.

A simple, purely algebraic derivation is given for the diagonal or near-diagonal canonical forms for the Lorentz-Hermitian matrix. (Received October 5, 1951.)

\section{A. S. Davis: The Euler-Fermat theorem for matrices.}

Let $A$ be a square matrix of order $n$ with integral rational elements whose determinant is relatively prime to the prime number $p$. J. B. Marshall (Proceedings of the Edinburgh Mathematical Society (2) vol. 6 (1939-1941) pp. 85-91) determined an exponent $q$ such that $A^{q} \equiv I(\bmod p)$ for every $A$, where $I$ is the unit matrix, and proved that for certain small values of $p$ and $n$ this exponent is the smallest possible. I. Niven (Duke Math. J. vol. 15 (1949) pp. 823-825) showed that Marshall's exponent is always the best possible. In this paper this problem is generalized for arbitrary moduli $m$. It is shown that an exponent $w$ exists such that $A^{w} \equiv I(\bmod m)$ for every $A$ and it is proved that $w$ is the best possible exponent. (Received July 17, 1951.)

\section{V. E. Dietrich: Algebras of finite projective planes from incidence matrices.}

For each ordering of lines of each of two pencils and points of the line of centers there is associated a unique standard incidence matrix. A block submatrix is designated the kernel. The elements of the kernel are order $n$ permutation matrices. Each element of the first column and first row of the kernel is $I_{n}$. Each of the remaining rows and columns, designated row sets and column sets, corresponds to a set of singly transitive permutations on $n$ letters. For a fixed $y$-axis, origin, and ideal line, every coordinatization of the affine plane possesses a ternary relation reducible to two binary relations, addition and multiplication, if and only if each column set of the corresponding kernel is isomorphic to the additive set which is then a group. Multiplication is right-distributive over addition if and only if the column sets are automorphisms of a group, which further implies that the additive group is abelian of type $(p, p, \cdots, p)$. Necessary and sufficient conditions on the kernel for commutativity, for associativity, and for left-distributivity of multiplication then imply the plane is Desarguesian. Using a result of Wedderburn's allows omission of either commutativity or left-distributivity. (Received October 8, 1951.)

\section{2t. J. C. Eaves: On the skeletons and characteristic roots of sets of matrices having a delayed commutativity property.}

If $A, B, C, \cdots$ is a finite set of $n \times n$ commutative matrices having elements in a field and having characteristic roots $\alpha_{i}, \beta_{i}, \gamma_{i}, \cdots(i=1,2, \cdots, n)$ respectively and if $f(x, y, z, \cdots)$ is any rational function, then by a well known theorem due to Frobenius the characteristic roots of the matrix $f(A, B, C, \cdots)$ are $f\left(\alpha_{i}, \beta_{i}, \gamma_{i}, \cdots\right)$ $(i=1,2, \cdots, n)$. This property of the characteristic roots is called the property "p." McCoy introduced a type of delayed commutativity in his quasi-commutative matrices which he showed also possess the "p" property. In this paper some pairs and some finite sets of matrices having a delayed type of commutativity and possessing 
finite chains of commutes are considered and are shown to have the " $p$ " property. A generalization is given for a finite set of quasi-commutative matrices. (Received October 10, 1951.)

43t. D. O. Ellis: On immediate inclusion in partially ordered sets and the construction of homology groups for metric lattices.

In this note conditions are found which characterize the immediate inclusion relations arising from partial orderings of a set among binary relations on the set. Characterizations of modularity and distributivity are given in lattices in terms of immediate inclusion. It is shown how the chains of elements of a given norm in a normed distributive lattice may be formed and the boundary operator mapping the group of $p$-chains onto a subgroup of the $q$-chains (the face relation being immediate precession) may be defined for $q<p$ in case the lattice has the finite ascending chain condition. An additional condition is imposed under which the $p$-bounding $q$-chains are $q$-cycles for the $r$-chains for $r<q<p$ so that homology groups $H_{p r}^{q}$ may be defined. The methods are elementary in nature. (Received September 10,1951.)

\section{D. O. Ellis: Remarks on isotopies.}

One considers property g. A. of a groupoid $G\left({ }^{*}\right)$, namely the possession of four permutations $P, Q, R$, and $S$ so that $x P^{*} y Q=y R^{*} x S$. One finds: (1) If $G\left(^{*}\right)$ has unit $e$ and is $g . A$., there is a permutation $V$ so that $x^{*} y=y V^{-1 *} x V$, the set $\left\{e V^{n}\right\}, n=0$, $\pm 1, \pm 2, \cdots$, is a subgroup of $G\left({ }^{*}\right)$, and if $e V$ is idempotent, $G\left(^{*}\right)$ is Abelian. (2) $A$ unit possessing semigroup which is $g$. A. is Abelian. (3) A quasigroup (finite groupoid with left and right nonsingular elements) is isotopic to an Abelian group (Abelian semigroup) if and only if it is g. A. and associative in the sense of Evans (J. London Math. Soc. vol. 25 (1950) pp. 196-201). It is also shown that: (4) Tro semilattices are isotopic if and only if they are isomorphic. (5) $A$ semilattice admits a second operation to form a lattice if and only if it is isotopic to a semilattice of a lattice in which case the two lattices are isomorphic. (Received August 6, 1951.)

\section{Wallace Givens: Fields of values of a matrix.}

Given a square matrix $A$ and a positive definite Hermitian matrix $H$, the field of values of $A$ with respect to $H, F_{H}(A)$, is the set of all complex numbers $(x, A x)_{H} /(x, x)_{H}$, where $x$ is an arbitrary nonzero vector and $(x, y)=x^{*} H y$ is the inner product in the metric defined by $H$. It is shown that: (1) $F_{H}(A)=F_{I}(B)$ where $I$ is the unit matrix and $B$ is a matrix similar to $A$; (2) if $A$ has a nonsimple elementary divisor with root $\lambda$, then $\lambda$ is an interior point of $F_{H}(A)$ for every $H$; (3) if $A$ and $H$ are simultaneously decomposable, $F_{H}(A)$ is the minimum convex set containing the component regions; (4) the intersection of the $F_{H}(A)$ for all $H$ is the minimum convex polygon $P(A)$ containing all the roots of $A$; (5) a necessary and sufficient condition for $F_{H}(A)=P(A)$ for some $H$ is that the elementary divisors corresponding to roots lying on the boundary of $P(A)$ shall all be simple. (Received October 11,1951.)

46. Emilie V. Haynsworth: Bounds for determinants with dominant main diagonal.

Let $D$ be a determinant of order $n$ for which the absolute value of each element $a_{i i}$ in the main diagonal is greater than or equal to the sum of the absolute values of all other elements $a_{i j}$ in the same row. In a large number of papers bounds have been obtained for such determinants. (For references to the literature on such determinants, 
see the paper by $\mathrm{O}$. Taussky, A recurring theorem on determinants, Amer. Math. Monthly vol. 56 (1949) pp. 672-676.) Recently G. B. Price, Proceedings of the American Mathematical Society vol. 2 (1951) pp. 497-502, has improved these results in the following manner: Set $d_{i}^{(k)}=\left|a_{i i}\right|-\sum_{j=1, j \neq i}^{k}\left|a_{i j}\right|$ and $S_{i}^{(k)}=\left|a_{i i}\right|$ $+\sum_{j=1, j \neq i}^{k}\left|a_{i j}\right|$. Then $|D| \geqq a_{11} \prod_{h=2}^{n} d_{h}^{(h)}$, and $|D| \leqq a_{11} \prod_{h=2}^{n} S_{h}^{(h)}$. In this paper the following lower bound is obtained: $|D| \geqq a_{11} \prod_{h=2}^{n} d_{h}^{(h)}+\sum_{i=2}^{n}\left[\left(\sum_{j<i}\left|a_{i j}\right|\right)\right.$ - $\left.\prod_{k=1}^{i=1} d_{k}^{(i)} \prod_{h=i+1}^{n} d_{h}^{(h)}\right]$. Similarly, a better upper bound is obtained. Moreover, some related theorems on determinants with relatively large main diagonals are proved, and applications made to the characteristic roots of a matrix. (Received October 8, 1951.)

\section{G. B. Huff: On matrices $A$ such that $A^{t}$ is a matric polynomial in $t$.}

If $A$ is a square matrix with elements from a field $F$ of complex numbers, there may exist a matric polynomial $F(X)$ such that $A^{t}=F(t)$ for every rational integer $t$. This paper studies the implications of the definition $A^{C}=F(C)$, where $A$ has the property described above and $C$ is a square matrix. It is shown that under appropriate conditions the usual laws hold and applications are indicated. (Received October 1, 1951.)

\section{R. J. Levit: The minimax solutions of a system of linear equa- tions.}

Let $S$ denote the totality of real solutions $x=\left(x_{1}, \cdots, x_{n}\right)$ of a consistent system of linear equations, $\sum_{j=1}^{n} a_{i j} x_{j}=b_{i}, i=1, \cdots, m$, with real coefficients. If $\max \left|x_{j}\right|$, $j=1, \cdots, n$, has a minimum value $\rho$ for $x$ in $S, \rho$ is called the minimax of $S$; and $x^{*}=\left(x_{1}^{*}, \cdots, x_{n}^{*}\right)$ is called a minimax solution of the system if $\max \left|x_{j}^{*}\right|=\rho$. In the case of a single equation, explicit expressions for $\rho$ and $x^{*}$ are known. (See H. Rademacher and I. Schoenberg, Canadian Journal of Mathematics vol. 2 (1950) pp. 251252.) In the present paper the existence of a minimax solution is established for the general linear system and explicit expressions for $\rho$ and $x^{*}$ are obtained. The result is then used to generalize some theorems of C. de la Vallee Poussin concerning best approximation to functions on finite sets. (See his monograph, Lecons sur approximation des fonctions d'une variable reelle, pp. 78-86, Paris, 1919.) (Received October 11, 1951.)

\section{Nathaniel Macon: On the approximation of irrational numbers.}

Let $\alpha$ be irrational and $t$ real. Denote by $x_{\min }(t)$ the smallest integer $x$ such that there exists an integer $y$ with $1 \leqq x \leqq t$ and $|\alpha x-y|<1 / t$. Denote the consecutive values of $x_{\min }(t)$ so obtained by $x_{0}=1<x_{1}<x_{2}<\cdots$ and the corresponding values of $y$ and $t$ accordingly. Koksma [Mathematica vol. $6(1938) \mathrm{pp} .113-143$ ] proved that at least one of the three inequalities $x_{i} / t_{i}>\left(4+\lambda^{2}\right)^{1 / 2}(i=n, n+1, n+2)$ holds for any $\lambda$ such that $\lambda_{n+2}=\left(x_{n+2}-x_{n}\right) / x_{n+1} \geqq \lambda$. He showed that a well known theorem of Borel [J. Math. Pures Appl. (5) vol. 9 (1903) pp. 329-375] for the convergents of the expansion of $\alpha$ as a regular continued fraction is contained in the case $\lambda=1$. It is proved here, without used of continued fractions, that either at least two of the inequalities $x_{i} / t_{i}$ $>5^{1 / 2}(i=n-2, n-1, \cdots, n+2)$ or else $x_{n} / t_{n}>3$. This is the analogue of a theorem of Brauer and Macon [Amer. J. Math. vol. 71 (1949) pp. 349-361] for the convergents of the continued fraction $\alpha$. (Received October 12,1951.) 
50. G. W. Medlin: Bounds for the imaginary characteristic roots of real matrices.

A. Brauer [Duke Math. J. vol. 14 (1947) pp. 21-26] has shown that each characteristic root of the matrix $A=\left(a_{k \lambda}\right)$ must lie in the interior or on the boundary of at least one of the $n(n-1) / 2$ ovals $\left|z-a_{\kappa k}\right| \cdot\left|z-a_{\lambda \lambda}\right| \leqq P_{k} P_{\lambda}$, where $P_{r}+\left|a_{r k}\right|$ is the sum of the absolute values of the elements of the $r$ th row. In a paper presented at the symposium of the Institute for Numerical Analysis at Los Angeles in August 1951, he obtained other ovals containing the roots which of ten are smaller. Moreover, he will present an improvement of this result for the real characteristic roots of real matrices at the meeting of the American Mathematical Society at Washington in October. It will be shown in this paper that one of Brauer's methods for obtaining bounds for the real roots can be modified so that new bounds are obtained for the imaginary roots of real matrices. These are often better than Brauer's bounds. (Received October 8, 1951.)

51. Benjamin Evans Mitchell: A canonical form for nonderogatory matrices under unitary transformation.

Techniques of the theory of groups with operators are applied to show that any complex matrix $A$ may be unitarily transformed to a triangular matrix with unique nonderogatory diagonal blocks $A_{1}, \cdots, A_{k}$ where $A_{i}$ has only one distinct characteristic root and has positive elements in the diagonal below the main diagonal. Moreover the Jordan normal form of $A$ is $A=B_{1}+\cdots+B_{k}$, where $A_{i}$ is similar to $B_{i}$ for $i=1, \cdots, k$. Let $A$ be unitarily equivalent to $T_{1}$ and also to $T_{2}$ where $T_{1}$ and $T_{2}$ are in the above triangular form and each has the same diagonal blocks $A_{1}, \cdots, A_{k}$. Then if no two $A_{i}$ have the same characteristic root, any unitary matrix $U$ such that $T_{1} U=U T_{2}$ is a diagonal matrix. Thus if $A$ is nonderogatory, the absolute value of all elements below the diagonal blocks in the above triangular form is invariant, and hence by a simple convention a unique triangular canonical form is given for a specified ordering of the characteristic roots. (Received October 5,1951 .)

\section{D. R. Morrison: Bi-regular rings and the ideal lattice isomor- phisms.}

Generalizing an observation of Foster, the set $R^{0}$ of idempotent elements in the center of a ring $R$ is a Boolean ring under a modified addition and ring multiplication. If $I$ is an ideal of $R$, then $I^{0}$ is an ideal of $R^{0}$. If $J$ is an ideal of $R^{0}$, then $R J$ is an ideal of $R$ and $(R J)^{0}=J$. A necessary and sufficient condition that $R I^{0}=I$ for each ideal $I$ of $R$ is that every principal ideal of $R$ have a unit element, or, in the terminology of Arens and Kaplansky, that $R$ be bi-regular. Thus in the bi-regular case, the (principal) ideal lattices of $R$ and $R^{0}$ are lattice-isomorphic. It follows that every theorem on Boolean rings which is expressible in terms of the ideal and principal-ideal lattices is extended immediately to bi-regular rings. A corollary is Arens and Kaplansky's extension to bi-regular rings of Stone's "mathematical equivalence" between Boolean rings and Boolean spaces. Relationships are established between the homomorphisms and subdirect sum representations of $R$ and of $R^{0}$. (Received October 11,1951.)

\section{Erna H. Pearson: On certain nonhomogeneous Diophantine} equations. Preliminary report.

The congruence $1+A X^{c} \equiv B Y^{v}(\bmod p)$, where $p$ is a prime of the form $1+v c$, 
and $(A B, p)=1$, is considered. If $g$ is a primitive root of $p$, this congruence may be written $1+g^{i} x^{c} \equiv g^{i} y^{v}(\bmod p)$, where $A \equiv g^{i+z c} ; B \equiv g^{j+u v} ; x \equiv g^{z} X ; y \equiv g^{u} Y ; 0 \leqq i<c$; and $0 \leqq j<v$. The author determines the number of solutions of the congruence for a given $i$ or $j$ when $(X Y, p)=1$, and the conditions on $i$ and $j$ for solutions when $(X Y, p)$ $=p$. If no solutions exist for a given $(i, j)$, and $(d, p)=1$, there are no integral solutions of the Diophantine equation $\left(d+k_{1} p\right)+\left(d g^{i}+k_{2} p\right) x^{c}-\left(d g^{j}+k_{3} p\right) y^{v}=0$. The author also carried out computations for certain primes of the above form with $v=5,7$, 11 , and 13 , and noted the number of solutions of the congruence for given $(i, j)$. (Received October 10, 1951.)

54. J. H. Wahab: Some new cases of irreducibility for Legendre polynomials.

Holt (Proc. London Math. Soc. vol. 11 (1912-1913) pp. 351-356 and vol. 12 (1913) pp. 126-132) proved the Legendre polynomial $P_{n}(x)$ of degree $n$ irreducible for $n=2^{m}, 2^{m}+1$, and for certain degrees near odd primes or twice odd primes. Ille (Thesis, Abstract in Jahrbuch der Dissertationen der Universität Berlin (1924)) established irreducibility for $n=(p-1) p^{k}$ ( $p$, odd prime) and completed Holt's proof that a pure quadratic factor can not exist. In this paper a theorem of Grosswald (Proceedings of the American Mathematical Society vol. 1 (1950) pp. 553-554) is used to prove: (1) If $P_{n}(x)$ is reducible and if $n$ and the degree of each factor are expressed as the sums of different powers of two, then each power of two used in the expression for $n$ will occur once, and only once, among the terms in the representations of the degrees of the factors; (2) Only polynomials in $x^{2}$ are possible factors; (3) $P_{n}(x)$ is irreducible for $n=t \cdot 2^{m}+0,1,2,3(t<19)$ and for some new cases near an odd prime or twice an odd prime; (4) There are only nine $P_{n}(x)(n \leqq 500)$ not known to be irreducible. (Received July $5,1951$. )

\section{R. A. Willoughby: p-algebras.}

A. L. Foster has studied $p$-rings in connection with his general $K$-ality theories and has shown that each $p$-ring is related to a certain " $p$-algebra" by an inter-equational bond which generalizes the Boolean $(p=2)$ case. The primitive operations of a $p$-algebra, realized in the (corresponding) $p$-ring, are $x,=$ the ring multiplication, and $\wedge$, the unary operation defined by $a^{\wedge}=1+a$. The purpose of this paper is to present a set of postulates for $p$-algebras and to study certain of their intrinsic properties. The postulates are essentially in equational form and, in fact, the equations satisfied over a general $p$-algebra are shown to be just those satisfied over the simplest case ( $=$ the $p$-algebra corresponding to the field of residues $\bmod p$ ). In this study the "normal representation theorem" discovered by Foster constitutes a basic tool. The initial additive form of this theorem is here shown to have a convenient multiplicative adaptation. A second important tool is developed in the form of certain cancellation and substitution laws. (Received October 10,1951.)

\section{ANALysis}

\section{J. W. Ellis: A general set-separation theorem.}

The result in this paper has as applications Stone's theorem on ideals in distributive lattices, Tukey's theorem on separation of convex sets, and a theorem of Klee's on cones in linear topological spaces. Let $\mathcal{S}$ denote the collection of all subsets of a set $S$. Let $\phi$ be a function on $\mathcal{S}$ to $\mathcal{S}$ satisfying: (i) for all $A, \phi(A) \supset A$; (ii) for all $A$, $\phi[\phi(A)] \subset \phi(A)$; (iii) $\phi(A)=\bigcup\{\phi(F): F$ finite, $F \subset A\}$; (iv) if $p \in S$ and $F$ is finite, 
$\phi(F \cup\{p\}) \subset \bigcup[\phi(\{a\} \cup\{p\}): a \in \phi(F)]$. Let $\psi$ be another function, also satisfying (i)-(iv), related to $\phi$ in such a way that, if $a \in \psi(\{b\} \cup\{c\})$ and $d \in \phi(\{e\} \cup\{c\})$, then $\phi(\{a\} \cup\{e\}) \cap \psi(\{b\} \cup\{d\}) \neq \Lambda$. Then if $\phi(A)=A$ and $\psi(B)=B$, and $A \cap B=\Lambda$, there are sets $A^{\prime} \supset A$ and $B^{\prime} \supset B$ such that $\phi\left(A^{\prime}\right)=A^{\prime}, \psi\left(B^{\prime}\right)=B^{\prime}, A^{\prime} \cap B=\Lambda$, and $A^{\prime} \cup B^{\prime}=S$. (Received October 11, 1951.)

\section{Tomlinson Fort: Reducibility of differential equations. Prelim- inary report.}

In this abstract consideration is given only to the third order equation. Generalization to the $n$th order equation will be given in the paper. The equation $y^{\prime \prime \prime}+p_{1}(x) y^{\prime \prime}$ $+p_{2}(x) y^{\prime}+p_{3}(x) y=0$ where $p_{1}(x), p_{2}(x), p_{3}(x)$ are continuous when $a \leqq x \leqq b$ is called reducible over this interval if it has two solutions $y_{1}$ and $y_{2}$ which constitute a fundamental system of solutions of an equation $y^{\prime \prime}+P_{1}(x) y^{\prime}+P_{2}(x) y=0$ where $P_{1}(x)$, $P_{2}(x)$ are continuous over $a \leqq x \leqq b$. Necessary and sufficient conditions for reducibility are obtained involving differential equations. Sufficient conditions are obtained involving $p_{1}(x), p_{2}(x), p_{3}(x)$ alone. (Received October 5, 1951.)

58t. R. E. Fullerton: On the rectification of contours of a Fréchet surface.

Let $S$ be a Fréchet surface with respresentation $T: x=x(w), w \in[0,1 ; 0,1]$ from $E_{2}$ to $E_{8}$ and let $f$ be a real-valued Lipschitzian function on $[S]$, the set of points occupied by $S$. Let the contour $\beta_{t}^{*}$ be the boundary of the set $\beta_{t}=E[w \in[0,1 ; 01] \mid f(x(w))<t]$. Let $\left\{t_{i}\right\}$ be any countable set of real numbers with $\min _{x} \in[S] f(x)<t_{i}<\max _{x} \in[S] f(x)$, $i=1,2, \cdots$, for which the length of each $\beta_{t i}^{*}$ is finite in the sense defined by Cesari (Bull. Amer. Math. Soc. Abstract 57-3-188). It is shown that there exists a mapping $T^{\prime}$ Fréchet equivalent to $T$ for which each $\beta_{t i}^{*}$ is a union of arcs the sum of whose lengths is finite. (Received October 8, 1951.)

59. J. J. Gergen and F. G. Dressel: Uniqueness for p-regular mapping.

Let $\Gamma$ be a circle about the origin, and let $S$ be the interior of $\Gamma$. Let $S^{\prime}$ be a finite domain whose boundary is a simple, closed, rectifiable curve. Let $p(x, y)$ be real, positive, and continuous on $S+\Gamma$, and have continuous bounded derivatives $p_{x}, p_{y}$ on $S$. In a previous paper (Duke Math. J. vol. 18 (1951) pp. 185-210) the authors obtained a generalization of the existence part of the Riemann mapping theorem for equations $p u_{x}=v_{y}, p u_{y}=-v_{x}$, and domains $S$ and $S^{\prime}$. In the present paper uniqueness results are obtained. (Received October 8, 1951.)

60. B. F. Hadnot: Note on a class of series.

Consider the series (1) $\sum_{n=1}^{\infty} c_{n} g(z+n) n^{-\mu_{1}^{z}}$, where for fixed $z, g(z+n) n^{-\mu_{1} z}$ has a determinate asymptotic representation by means of a power series in $1 / n$. For a particular class of functions $g(z)$ with the mentioned property for $g(z+n) n^{-\mu_{1} z}$, the author has established theorems relative to convergence, uniform convergence, and the analytic character of the function represented by the series (1). (Received October 10, 1951.)

61. E. P. Miles: Harmonic functions in 3-space generated by analytic functions of a hypervariable. Preliminary report.

Let $t=i x+j y+k z$ where $i^{2}=i, i j=j i=j, i k=k i=k, j^{2}=k$, and $j k=k j=-i$. A 
function $f(t)=i U(x, y, z)+j V(x, y, z)+k W(x, y, z)$ is said to be an analytic function of the hypervariable $t$ provided $U_{x}=V_{y}=W_{z}, U_{y}=V_{z}=-W_{x}$, and $U_{z}=-V_{x}=-W_{y}$. The components $U, V$, and $W$ satisfy $\nabla^{2} U=-\nabla^{2} V=\nabla^{2} W$; hence $U+V, V+W$, and their difference $U-W$ are harmonic. The functions $(i+j) f(t),(j+k) f(t)$, and $(k-i) f(t)$ where $f(t)$ is analytic have harmonic components. $t^{n}$ where $n$ is a positive integer is analytic with derivative $n t^{n-1}$. The ordinary sum, product, and quotient laws for differentiation hold, where quotients are defined with the aid of a multiplicative conjugate and a generalized absolute value. The conjugate for $t, i=i\left(x^{2}+y z\right)$ $+j\left(-z^{2}-x y\right)+k\left(y^{2}-x z\right)$, and the generalized absolute value, $|t|=i\left(x^{3}-y^{3}+z^{3}\right.$ $+3 x y z)^{1 / 3}$, satisfy the equations $t \bar{t}=|t|^{3},|\bar{t}|=|t|^{2},(\bar{t})=|t|^{3} t$ and $|t|^{3}=(x-y+z)$ $\cdot\left(x^{2}+y^{2}+z^{2}+x y-x z+y z\right)$. The function $1 / t=\bar{t} /|t|^{3}$ is analytic except at points of the plane $x-y+z=0$ and the normal to this plane at the origin. Analytic functions of a complex variable of the exponential and trigonometric type expandable in a McLaurin's series are readily generalized to analytic functions of $t$. (Received October 8, 1951.)

\section{T. A. Newton: Application of extended summation by parts.}

An extended summation by parts formula is developed by considering the expres sion $\sum a_{i} \Delta^{n} b_{i}$. A particular transformation for power series is then obtained which in many cases gives a closed expression for the sum of a power series. Applying this formula to the series $\sum_{i=0}^{\infty} i^{(m)} z^{i}$ yields properties of this series that are analogous to certain properties of the gamma function. Also, it is found that Euler's transformation is a particular case of this transformation for power series. (Received October 10, 1951.)

\section{3t. B. J. Pettis: Certain subalgebras of Boolean algebras.}

This paper is concerned with the relations between certain subalgebras generated by an arbitrary subset $E$ of a Boolean algebra $A$. By means of a theorem involving an abstract space admitting a family of binary operations, previous results due to Sierpinski, Saks, von Neumann, and Ambrose concerning representations for the $\sigma$-ring generated by $E$ are obtained and in some cases improved. (Received October 12, 1951.)

\section{J. W. Young: Nonclassical orthogonal polynomials.}

Investigation is made of the conditions under which the class of second order homogeneous linear differential equations $\left(A_{1} x^{r+2}+A_{2} x^{x^{+2}}\right) y_{n}^{\prime \prime}+\left(B_{1} x^{r+1}+B_{2} x^{s+1}\right) y_{n}^{\prime}$ $+\left[L_{1}(n) x^{r}+L_{2}(n) x^{8}\right] y_{n}=0$ possesses orthogonal polynomial solutions. It is shown that the classical orthogonal polynomials, such as those of Legendre, Hermite, and Laguerre, are contained as special cases. Particular attention is given to the nonclassical orthogonal polynomial solutions obtained when $L_{1}(n)=K_{1} L(n), L_{2}(n)$ $=K_{2} L(n)$. It is found that there are two general types of such solutions, one of order and subdegree linear in $n$, and having an algebraic weight function and finite interval of orthogonality; the other of order quadratic in $n$, and subdegree linear in $n$, and having a transcendental weight function and semi-infinite interval of orthogonality. With suitable restrictions, derivative definitions and integrated square formulas are obtained for these solutions. Certain other properties of interest in these solutions are also considered. (Received October 11, 1951.)

\section{Applied Mathematics}

65. O. R. Ainsworth: Asymptotic evaluation of displacements in one 


\section{elastic medium due to a $P$-wave point source in a second elastic medium.}

A $P$-wave point source is located $(0,0, a)$ in an isotropic, elastic, medium occupying $z>0$. A second medium occupies $z<0$, and is contiguous to the first medium along $z=0$. The displacements due to this source are computed asymptotically when the point of observation is far removed from the origin and is in the second medium at a distance of the order of $a$. (Received October 9, 1951.)

66. Ernest Ikenberry: On the significance of the arbitrary parameters entering into a Bruns' series expansion of the distribution function in phase space. Preliminary report.

The parameters $h_{i}(q, t)$ in the Hermite polynomials $H_{n}\left(h_{i} p_{i}\right)$ in a Bruns' series expansion of $f(q, p, t)$ are of no physical significance, since $\partial f / \partial h_{i}=0$, the distribution function being independent of any particular mode of representation ( $\mathrm{J}$. of Chem. Phys. vol. 19 (1951) pp. 467-470). From the properties of the operator $D$ and the Liouville equation $D f=0$ there is readily obtained, for any suitable function $Q(q, p, t)$, $D(f Q)=f D Q+Q D f=f D Q$. Likewise, $D_{h}(f Q)=f D_{h} Q$, where the operator $D_{h}$ is defined by $D=D_{h}+\sum_{i}\left(D h_{i}\right) \partial / \partial h_{i}$. The infinite set of partial differential equations which the coefficients in the Bruns' series satisfy are obtainable by choosing $Q$ 's which are products of Hermite polynomials $H_{n}\left(h_{i} p_{i}\right)$ and integrating the two members of the preceding relation over momentum space. These coefficients are functions of the $h_{i}(q, t)$, but the preceding relation shows that the partial differential equations are formally independent of the manner in which the $h_{i}$ depend upon the position coordinates and the time. In solving the partial differential equations, there is no loss of generality in treating the $h_{i}$ as constants. (Received October 8, 1951.)

\section{Geometry}

\section{Patrick Du Val: Loci whose curve sections are hyperelliptic.}

This study is of the algebraic $k$-fold ${ }^{\pi} H_{k}^{n}$ of order $n$, not generated by $\infty^{\prime}[k]_{\mathrm{s}}$, whose general curve section is hyperelliptic and of genus $\pi$ (expanding classical work of Castelnuovo, Rend. Circ. Mat. Palermo vol. 4 (1890), and Enriques, Rend. Accad. Lincei vol. 2 (1893), on the cases $k=2,3$ respectively). ${ }^{\pi} H_{k}^{n}$ is rational, and normal in $[n-\pi+k-1]$, and has a unique pencil of $(k-1)$-dimensional quadrics, tracing the canonical involution on the general curve section; the ambient $[k]_{\mathrm{s}}$ of these generate a normal $(k+1)$-fold of order $n-\pi+1$, of which ${ }^{\pi} H_{k}^{n}$ is the residual section by a quadric through $n-2 \pi-2[k] \mathrm{s}$ (or for $n<2 \pi+2$, is coresidual to a quadric section plus $2 \pi+2-n[k] \mathrm{s})$. If there is a point common to all quadrics of the pencil, this is a $(\pi+1)$-ple point of ${ }^{\pi} H_{k}^{n}$. The necessary and sufficient condition for ${ }^{\pi} H_{k}^{n}$ to be the projection of ${ }^{\pi} H_{k}^{n+1}$ from a simple point of itself is that a quadric of the pencil breaks up into a pair of $[k-1] \mathrm{s}$. If ${ }^{\pi} H_{k}^{n}$ is not a cone, then either $n \leqq 2 \pi+2$, or $n=2 \pi+3, k \leqq \pi+4$, or $n+2 k \leqq 4 \pi+8$. (Received October 9, 1951.)

\section{Benjamin Ernest Mitchell: Realization of the quaternion.}

The quaternion, being a four-dimensional concept, transcends intuitional perception. However, four perfectly good "views" may be had of it in four mutually orthogonal planes after the manner of the descriptive geometry. Furthermore, one may consider two of these four planes Gaussian and thus achieve an analytical realization of the quaternion through a one-to-one lineo-linear transformation of either of these planes into the other. And finally the quaternion is co-dimensional with the line joining two points in the complex projective plane which in turn at the hand of 
Laguerre has received intuitive representation in an ordered real point-pair. (Received October 8, 1951.)

\title{
Statistics and Probability
}

\author{
69. A. C. Cohen: The truncated Poisson distribution. Preliminary \\ report.
}

Consider a random sample from a Poisson frequency distribution, $f(x)=e^{-m} m^{x} / x !$, which is truncated at known terminal points. Using the principle of maximum likelihood, equations are derived for estimating the parameter $m$, both (1) when the number of unmeasured (missing) observations is unknown and (2) when the number of unmeasured observations is known. The results obtained are applicable to samples from which either one or both tails have been truncated. (Received October 10,1951.)

\section{TOPOLOGY}

\section{Haskell Cohen: Codimension. I.}

Let $X$ be a compact space, and let $G$ be the coefficient group for the AlexanderKolmogoroff cohomology groups. $\operatorname{Cd}(X)$, the codimension of $X$, is defined to be the greatest lower bound of those integers $n$ such that every element of $H^{n}(A)$ is extendable to $H^{n}(X)$ for each closed subset $A$ in $X$. It is shown that the sum theorem for closed sets holds, and also that $\operatorname{cd}(X \times I)=1+\operatorname{cd}(X)$. The definition is extended to include the codimension of locally compact spaces and the codimension of the pair $\left(X, X_{0}\right)$ where $X_{0}$ is a closed subset of $X$. It is shown that the theorems mentioned above hold in the latter two cases as well. (Received October 10,1951.)

\section{1t. S. T. Hu: On finite-dimensional compact connected groups.}

Let $G$ be a finite-dimensional compact connected group and $G_{0}$ a closed connected subgroup of $G$. It is well known that there is a totally disconnected closed central normal subgroup $Z$ of $G$ such that the quotient group $M=G / Z$ is a Lie group. Let $h: G \rightarrow M$ denote the natural projection and let $M_{0}=h\left(G_{0}\right)$. The main theorem of the paper states that the Cech cohomology ring $H\left(G / G_{0}\right)$ of the homogeneous space $G / G_{0}$ with real coefficients is isomorphic with $H\left(M / M_{0}\right)$ of the homogeneous space $M / M_{0}$ of the compact connected Lie group $M$ over its closed connected Lie subgroup $M_{0}$. In particular, if $G_{0}$ reduces to the neutral element $e$ of $G$, then $H(G) \approx H(M)$. Numerous applications are given in the paper; most of them are the generalizations of the theorems proved for compact connected Lie groups by $\mathrm{H}$. Hopf and his school. (Received September 21, 1951.)

\section{W. L. Strother: Fixed point theorems for multi-valued functions.}

This paper gives a definition of continuity and two fixed-point theorems for multi-valued functions. Theorem one states that every continuous multi-valued function on the closed unit interval to itself has a fixed point. Theorem two states that every multi-valued function on a Tychonoff cube to itself which is continuous and satisfies one additional condition has a fixed point. Whether the product of spaces with the fixed point property for single-valued functions necessarily has the fixed point property is an open question. Theorem one above is used to answer this in the negative for multi-valued functions. (Received October 10, 1951.) 


\section{3t. A. D. Wallace: $A n$ addition theorem for maps.}

Special cases of the following result are known. Let $X=X_{1} \cup X_{2}, A=X_{1} \cap X_{2}$, where $X_{1}$ and $X_{2}$ are closed. Let $f_{i}:(X, A) \rightarrow(Y, B)$ be maps $(i=1,2)$ such that $f_{1}\left(X_{2}\right) \subset B \supset f_{2}\left(X_{1}\right)$ and $f_{1}\left|A=f_{2}\right| A$. Define $f_{1} \bigvee f_{2}$ by $\left(f_{1} \bigvee f_{2}\right)\left|X_{1}=f_{1}\right| X_{1}$ and $\left(f_{1} \bigvee f_{2}\right) \mid X_{2}$ $=f_{2} \mid X_{2}$. Then $f_{1} \bigvee f_{2}:(X, A) \rightarrow(Y, B)$ is a map such that $\left(f_{1} \bigvee f_{2}\right)^{*}=f_{1}^{*}+f_{2}^{*}$ if $X$ is fully normal or $X$ is locally compact Hausdorff and $F(A)$ is compact. Here the ${ }^{*}$ denotes the induced homomorphism of the Alexander-Kolmogoroff cohomology groups. Suppose (for example) that $Y$ is a group and that $B$ is the identity element. Then $\left(f_{1} \cdot f_{2}\right)^{*}=f_{1}^{*}+f_{2}^{*}$. This is the analogue for cohomology of the commutativity of the homotopy groups of a group. (Received October 10,1951.)

\section{C. T. Yang: Open maps and homomorphisms.}

Floyd [Ann. of Math. vol. 51 (1950) p. 573] characterized light open maps as follows: Let $X$ and $Y$ be connected, locally connected compacta and let $f$ be a light map of $X$ onto $Y$, then $f$ is open if and only if for any connected open set $V$ in $Y$ and any component $U$ in $f^{-1}(V), f^{*}: H^{\prime}(\bar{V}, \bar{V} / V) \rightarrow H^{\prime}(\bar{U}, \bar{U} / U)$ is an isomorphism into, where the cohomology groups are the Cech groups with integral coefficients. By means of the construction of the Alexander-Kolmogoroff groups a direct proof of this characterization is given without involving the homotopy classes of maps into the unit circle and the result is slightly generalized. For instance, the cohomology groups can be taken as the Alexander-Kolmogoroff groups with coefficients in a nontrivial additive abelian group without torsion and either (i) compact Hausdorff spaces replace compacta or (ii) delete the compactness of $X$ and $Y$ and let $f$ be closed. Further, the dual of a theorem of Whyburn [Duke Math. J. vol. 4 (1938) p. 8] follows as a corollary. (Received October 10, 1951.) 